\title{
Routing Schemes in Software-defined Vehicular Networks: Design, Open Issues and Challenges
}

\author{
Liang Zhao, Ahmed Al-Dubai, Albert Y. Zomaya, Geyong Min, Ammar Hawbani, and Jiajia Li
}

\begin{abstract}
Software-defined vehicular networks (SDVN) is a promising technology to overcome the limitations of current vehicular networking. However, existing vehicular routing schemes are not equipped to handle communication in SDVNs. In addition, routing schemes in SDVNs, in general, has been lightly addressed in the literature. To fill this gap, this article explores the potential of SDVNs from the aspect of routing and studies the design principles of routing schemes in SDVNs and classifies the current routing solutions based on different criteria. SDVN routing schemes are then compared through comprehensive analysis, and key open issues and opportunities for future research directions are discussed.
\end{abstract}

\section{Introduction}

Vehicular communication plays an essential role in enabling a myriad of applications, such as traffic control, collision avoidance, and lane change assistance, in intelligent transportation system. This communication paradigm paves the way for many applications to realize the future in ultimately autonomous driving. Indeed, efficient vehicular networking is fundamental to a wide range of applications under the umbrella of road safety, business and entertainment.

The major research trend in the network layer of vehicular communication is under the umbrella of vehicular ad hoc networks (VANETs). VANETs become prominent when messages need to be relayed among vehicles and infrastructure in a multihop fashion. In particular, VANETs can even be deployed with fewer infrastructures to facilitate cost-effective vehicular communication. Routing algorithms are deployed to select the messageforwarding path with/without monitoring network traffic and are intended to provide efficient transmission. However, the current networking schemes cannot satisfy the increasing demand for transmission efficiency and massive amount of data where the deployment and network management of such a network is difficult to achieve. The general reason behind this is, for example, routing schemes are hardly upgraded in the fixed network devices under the current distributed routing architecture.
Thus, in respect of routing techniques, we summarize the following challenges of traditional VANETs.

Scalability and Adaptability: The current routing algorithms lack scalable properties in VANETs [1]. This is due to the fact that most existing studies consider either urban scenarios or highway scenarios. One routing algorithm cannot fit all scenarios because of the simplicity requirement of the designing protocols in traditional networks and due to the limited computation power of on-board network devices.

Efficient Routing Metrics: The design of routing metrics in traditional VANETs is similar to other forms of mobile ad hoc networks while only a limited number of factors are considered in route selection such as traffic lights, intersections, direction or speed and so forth. In fact, in contrast to other mobile ad hoc networks, there is a large number of routing related factors including ones mentioned above as well as, for instance, weather conditions, driver profile and behavior, road traffic, vehicle related information and so on. However, it is still vital to consider the appropriate factors in order to deploy and orchestrate the most appropriate routing metrics while the computation power of the network device is not sufficient for collecting and fusing data.

Quality of Services: Providing connectivity to all vehicles on the road could generate huge amounts of network traffic. Although facilitating different vehicular communication based services with different levels of requirements can be a solution, there are many exception cases. For example, the communication quality could be ensured due to the massive number of unmanaged data messages while different emergency messages are spread among neighboring vehicles. This is a key drawback of the distributed computing fashion of ad hoc networks that lack centralized management to control congestion and priority.

Global Information Management: The majority of research [2] focuses on how to find efficient data transmission routes on the basis of grasping the location or other information of the destination vehicle node. In fact, this research did not explicitly provide such a mechanism of requesting data. Although later studies provide some solutions to distribute the vehicle status data, these mechanisms show low efficiency as they could generate huge amount of overhead [1][2].

In the light of the aforementioned, it is clear that existing 


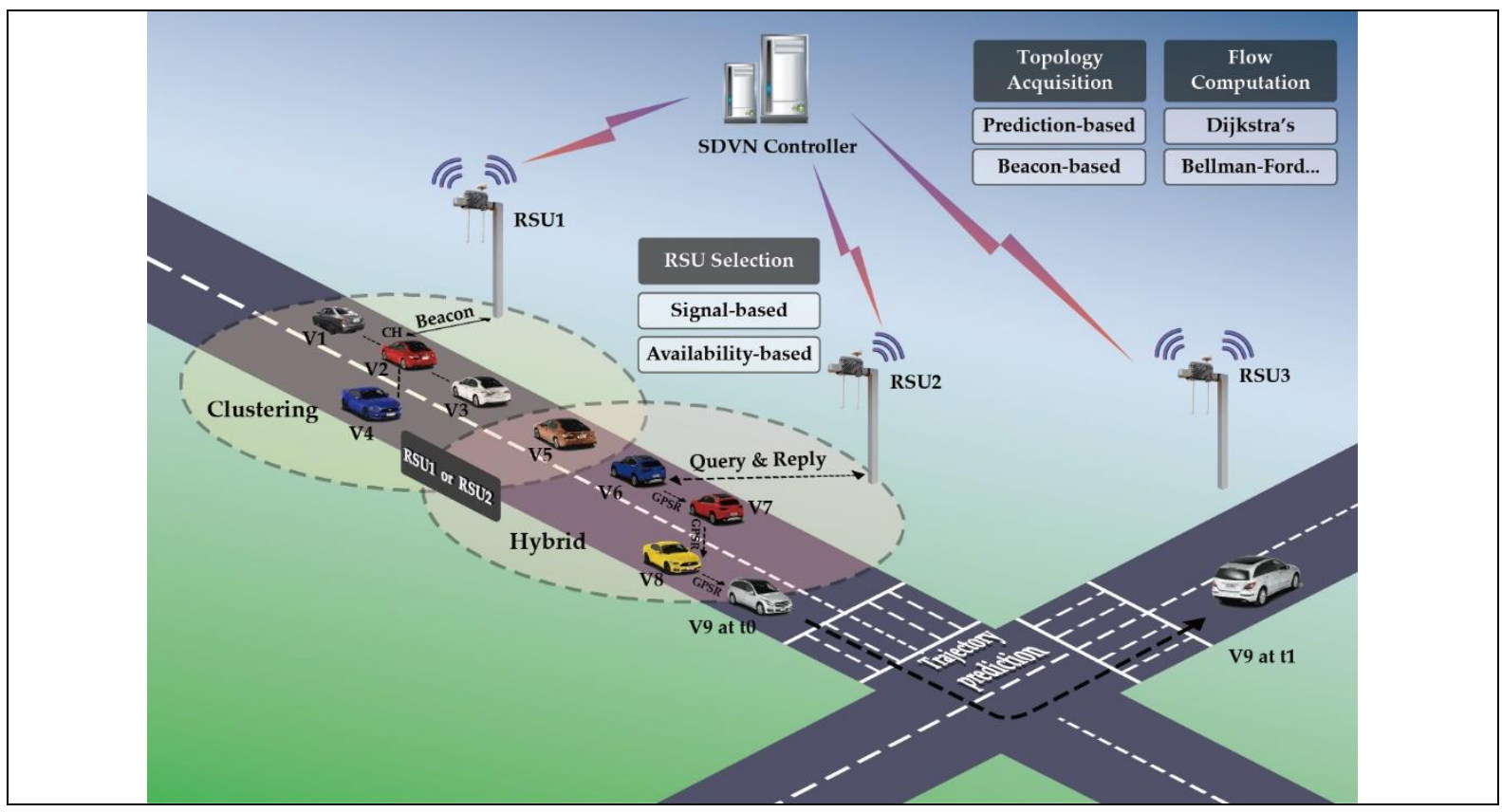

Figure 1. The basic principle of routing in SDVN.

solutions are not equipped to overcome the technical challenge using VANETs. Providing efficient transmission under different traffic conditions to satisfy all the requirements of applications will be challenging with that approach. The rigidity of underlying infrastructure offers few options for protocols innovation or improvement. Indeed, this will create a barrier for the practical deployments of novel vehicular networking solutions.

In order to realize the applications of connected vehicles and future autonomous driving, it is a vital to find a reasonable routing solution which must be scalable, adaptive, flexible and efficient. To this end, as the most promising technology in networking, software-defined vehicular networks (SDVN), is a potential candidate for orchestrating vehicular communication where it enables the programmability and separates the control and data from the conventional five-layer architectures. By decoupling the control plane from the data and application planes, it simply provides the network management and utilizes the traffic flows in a more efficient manner based on the real needs of applications. Thus, with the centralized controller, more proper routing paths can be calculated due to the benefits provided by SDVN such as high computation power, easier collection and management of routing relevant data, global view decision making and so forth. SDVN has great potential to mitigate the current barriers and restrictions that traditional VANETs and other forms of vehicular communication technologies encounter. Although it may create new or augment existing issues such as high overhead, desired enhancements can be made by applying the SDVN concepts which can enable better communications that support applications of autonomous driving such as Highway platooning and highway chauffeur.

This article investigates the routing related issues in SDVNs and presents a comprehensive overview of the state-of-the-art architectures, protocols, challenges, and potential solutions. In particular, this work aims to explore, analyze and classify the state-of-the-art, crossing high level goals and objectives towards detailed solutions such as those related to integrating existing routing techniques to the SDVN. This article places great emphasis on surveying the current routing technologies of multihop SDVNs, and discussing the potential of such networks. The future study of 5G-SDVN and C-V2X (Cellular based Vehicle-toEverything) could also benefit from the discussion outcomes of our work as both multihop-SDVN and C-V2X would be combined.

\section{Basic Principle of Routing in SDVN}

For the routing schemes in VANETs, the procedures of routing include node-status exchanging, route selection, and route maintenance and repair. Compared to the procedures of conventional routing schemes, the routing in SDVNs is similar but a little more complex, including association, network topology gathering, routing mode selection, and route selection (computation of flow table) and so forth. Therefore, before further revealing the classifications and challenges, we would like to present the common routing procedures through discussing the existing routing schemes.

As shown in Fig. 1, vehicles select the most appropriate RSU (road side unit) or gateway to send the status beacon and routing query. To manage the network flow, in each region, the controller needs to acquire the topology information to build the network graph from the path/link prediction of vehicles or beacon messages from the vehicles. By knowing the full/partial topology of the network, the controller then can maintain the traffic flow, respond to the routing query or distribute routing policy (hybrid SDVN). Finally, when the routing process is operated by a central or local controller, the computation of routes is achieved by running one of a variety of shortest path algorithms. 
The routing in SDVN operates based on the following detailed principles.

Vehicle Needs to Send Association Requests to Select A RSU to Connect in Order to Access the Controller: It is possible to have multiple candidate RSUs within the transmission range of a vehicle which generates additional queries for routing. During the routing phase, the selection of RSU for accessing the controller directly affects the efficiency of the routing algorithm. The optimal RSU is normally one with low traffic load and good communication signal. The RSU can be selected by iterating over a set of viable RSUs [3]. To further reduce the number of transmission requests, a RSU with a higher probability of receiving data is selected. Different methods for measuring the quality of RSU connections, including Nakagami fading model [4] and Kriging weights [5], are applied for this purpose. For example, in Fig. 1, V5 could choose the optimal RSU from either RSU1 or RSU2 to connect based on different measurement methods.

Appointed Controller Knows the Dynamic Changing of the Network Topology: The topology is constructed in the controller based on the periodic beacons from vehicle or link/trajectory prediction. If the periodic beacon is applied, vehicles send the beacon message including the velocity, location, direction or the status of its vehicle clusters and so on, to the controller, for example V2 acts as cluster head $(\mathrm{CH})$ in Fig. 1. The cluster information with the relative mobility table of all neighbor vehicles can also be encapsulated in the status beacon which enables the construction of the connectivity graph model in the controller. The destination of the beacon messages depends on the architecture of the SDVN. If the architecture is fully centralized, all beacons are sent to the central controller to draw the network topology. However, for a hierarchical architecture [4][6], the RSU acts as a local controller to maintain the network topology by receiving the beacon messages from local vehicles. The whole network can be partitioned into zones, where in each zone the local controller exchanges and updates the regional topology to the main controller. Then the main controller constructs the global topology based on the data from RSUs. Compared with the periodical beacon mode, the link prediction mode constructs the network graph on the partial collected data and trajectory prediction-based links [6] [7] which reduce the uplink overhead. With the advantages of trajectory prediction, the controller can infer the future topology in a short period of time. Then the controller can predict the future data transmission route, even with the broken links at the beginning. For example, in Fig. 1, based on the historical trajectory, surrounding vehicles or road traffic status, the controller predicts that V9 moves from the communication range of RSU2 to RSU3.

The Route of a Data Message Is Organized Centrally or
Hybrid According to Different Types of Routing Operations: In SDVN, the routes can be computed either in the controller (centralized mode), or in a hybrid mode of adhoc routing and controller-assisted routing (e.g. V6 sends data messages to V9 in ad hoc mode in Fig. 1). In centralized mode, upon receiving a route query from the source vehicle, the controller with the knowledge of the network topology takes complete responsibility for computing the entire path from source to destination [8]. In hybrid mode, the SDVN controller, as an instructor, draws the routing policy [9], defining the general routing behaviors and distributing the rules to the RSU or vehicle nodes, while RSU or vehicles use their local intelligence for forwarding data messages. If the network is hierarchical, the RSU that acts as the local controller is responsible for dealing with routing queries within its transmission range. If the local controller cannot find a route, the query then can be broadcast among the local controllers until finding one that is responsible for that destination vehicle. In hybrid mode [3] [10], vehicle clustering is widely used as a bottom layer to manage the local requests. The members of a cluster are computed by the controller, for example the neighbor nodes temporally sharing a common trajectory form a cluster [11]. As the member list is received by the vehicles, the most stable $\mathrm{CH}$ is selected based on the level of service quality in the cluster, for instance if the speed of the vehicle is close to the average speed of other vehicles in the cluster and it has reliable wireless transmission (better signal-to-noise ratio). In addition, a dual $\mathrm{CH}$ can be selected as a backup to avoid the potential risk when the primary $\mathrm{CH}$ fails, for example $\mathrm{V} 1$ can be the alternate $\mathrm{CH}$ in Fig. 1. Normally, the $\mathrm{CH}$ has two main duties: data collecting and handling local routing. First, the $\mathrm{CH}$ collects the status information of all vehicles within the cluster including position, velocity, direction and so forth by exchanging hello messages and reports the cluster status information to the controller. Second, the $\mathrm{CH}$ may act as a local controller to manage the inner cluster routing. As another derivation of hybrid mode [9] [12], the controller can also provide the routing tables for a short-term time horizon to vehicles. Upon receiving these routing tables, the vehicles run the active routing protocols in a distributed fashion by applying the pre-processed link-state information to generate routes.

Controller Computes the Routes Where the Weights of Links Are Calculated Based on Routing Metrics: The network topology is constructed by considering the quality of links. Routing metrics are used to measure the weight of a link which is the most important issue for shortest path planning in static graph as well as for vehicular communication. The shortest path algorithms, for example, Dijkstra [4], Eppstein's K-shortest [13], Bellman-Ford and Floyd algorithm [10], are applied while the route is calculated on the basis of a static graph constructed from the status data 


\begin{tabular}{|l|l|l|l|}
\hline \multicolumn{2}{|c|}{ Classification } & Advantages & Disadvantages \\
\hline \multirow{2}{*}{$\mathbf{2}$} & A. Centralized & Efficient route, high data delivery & Burden of central controller \\
\cline { 2 - 4 } & B. Hybrid & Low uplink overhead in central controller, load balancing & Low data delivery, high local overhead \\
\cline { 2 - 4 } & A. Single-path & $\begin{array}{l}\text { Implementation simplicity, good for low traffic or sparse } \\
\text { network }\end{array}$ & High latency, low throughput \\
\cline { 2 - 4 } & B. Multipath & Guaranteed data delivery, load balancing, short latency & Only work for dense network \\
\hline \multirow{2}{*}{3} & A. Beacon-based & Efficient route, high data delivery & High uplink overhead \\
\cline { 2 - 4 } & B. Prediction-based & Low overhead, scalable, good for dense network & $\begin{array}{l}\text { High latency due to complex prediction al- } \\
\text { gorithm }\end{array}$ \\
\hline \multicolumn{2}{|l}{ Table 1. Comparison of the advantages and disadvantages of subclasses of routing schemes in SDVNs. } \\
\hline
\end{tabular}

of the previous timeslot. To further guarantee the delivery of data message, multiple paths [4] [13] could be generated per source-destination pair in the controller. This approach intends to minimize the fallbacks of using static graph in real dynamic vehicular networks. In other words, the valid time of routes calculated based on static graph may not be enough to transmit data messages.

\section{Classifications}

This section categorizes the different routing schemes currently proposed. Since in SDVN most existing routing schemes are designed for unicasting, we only classify the unicast routing schemes in this article.

Centralized and Hybrid: For centralized routing schemes [4] [7] [8], the controller provides per-flow routes to vehicles which are more efficient for data message transmission based on the computation of the global-view controller. In contrast, hybrid routing schemes eliminate the burden of a central controller by moving the regional routing duty from central controller to local controller [3] [5]. Thus, we can classify SDVN routing schemes into centralized routing and hybrid routing.

Single-path and Multipath: In most studies [3] [5], the controller computes only one path (for unicast). However, due to the high dynamic nature of SDVN, links may be valid for a very short time which could be insufficient to accomplish the transmission of the required amount of data. Therefore, some schemes [13] establish multiple routes to guarantee the delivery of data messages. Hence, based on the number of paths used for data transmission, we can classify the existing SDVN routing schemes into

\begin{tabular}{|c|c|c|c|c|c|c|}
\hline Routing Scheme & $1 \mathrm{~A}$ & 1B & $2 \mathrm{~A}$ & $2 \mathrm{~B}$ & $3 \mathrm{~A}$ & 3B \\
\hline CESDT [7] & Yes & No & Yes & No & No & Yes \\
\hline HSDV [3] & No & Yes & Yes & No & Yes & No \\
\hline E-HSDV [3] & No & Yes & Yes & No & Yes & No \\
\hline Bozkaya [5] & No & Yes & Yes & No & Yes & No \\
\hline MOT [4] & Yes & No & Yes & No & No & Yes \\
\hline LDPR [13] & Yes & No & No & Yes & Yes & No \\
\hline HSAW [8] & Yes & No & Yes & No & Yes & No \\
\hline SVAO [10] & No & Yes & Yes & No & Yes & No \\
\hline Geospray [12] & No & Yes & No & Yes & Yes & No \\
\hline Pretti [6] & Yes & No & Yes & No & No & Yes \\
\hline
\end{tabular}

single-path routing and multipath routing.

Beacon-based and Prediction-based: The controller has to know the dynamic topology of the network. Thus, the update of the network topology can be processed in two modes, beacon-based mode or prediction-based mode. All vehicle status information is collected by receiving beacons sent by vehicles in beacon-based mode [3] [5]. In contrast, the controller only collects a limited number of beacons from vehicles and predicts the link status based on the historical data [7] [11]. Therefore, based on the approach of network topology maintenance, we can classify the existing SDVN routing schemes into beacon-based routing and prediction-based routing, where one particular routing scheme can fall into multiple subclasses.

\section{Comparisons}

We compare the routing approaches from a variety of perspectives in Tables 1, 2 and 3, respectively. We first compare the advantages and disadvantages among different subclasses in Table 1. Then we elaborate the classification of SDVN routing schemes in Tables 2 and 3. We further present a qualitative comparison as given below.

Complexity: This is defined by how much computation power (additional hardware) is required and how difficult it is to implement and execute a SDVN routing scheme. All prediction-based routing schemes [4] [7] [11] [14] are complex because the trajectory prediction has high complexity. In addition, running time-dependent routing [7] in a controller is complex due to its high computation requirement. Communication Overhead: This is defined as how many 


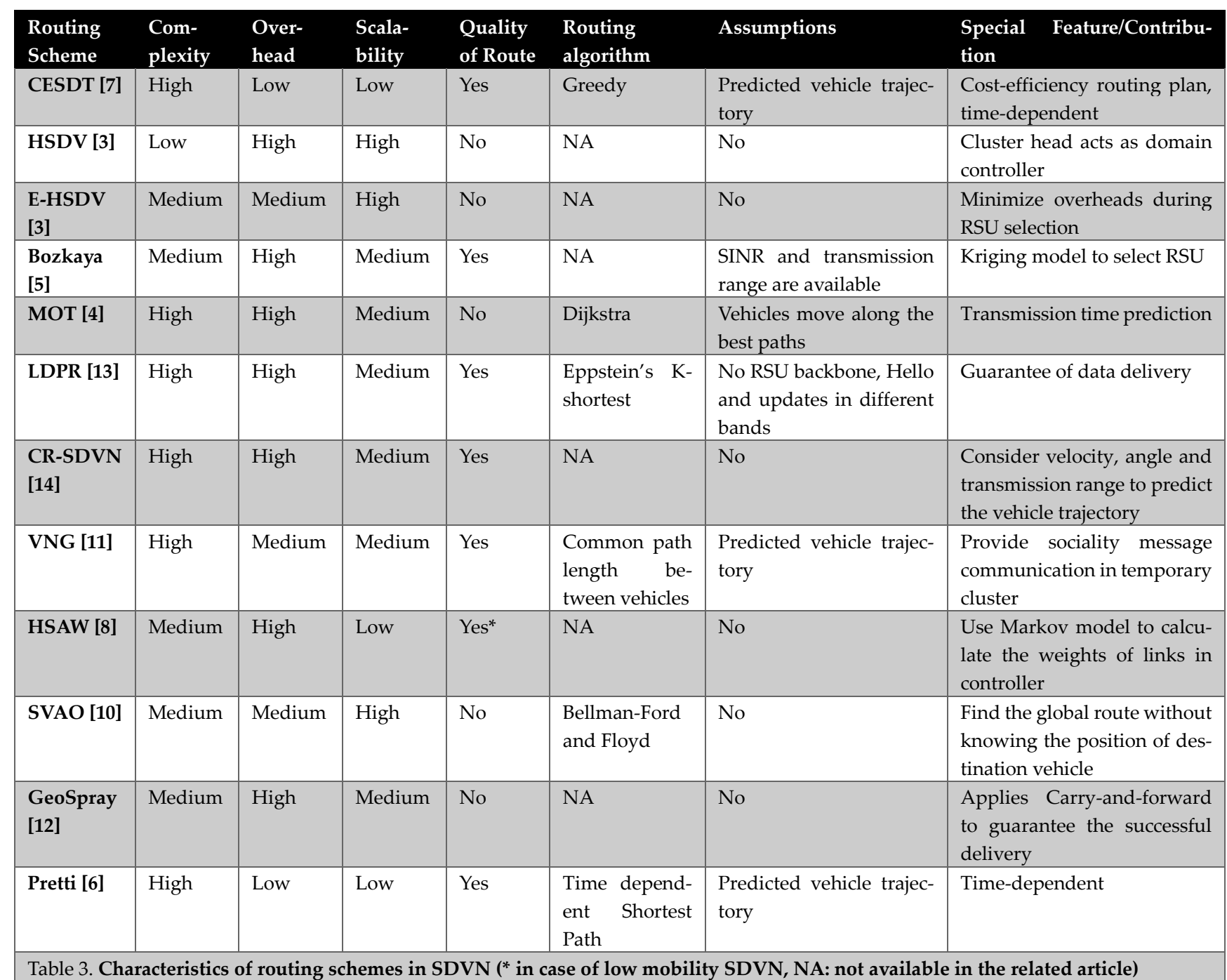

additional messages including status beacons, inner cluster messages, routing queries and replies and so on there are in SDVN. In particular, the application of trajectory prediction can reduce the number of status beacons while more routing queries can be generated locally in hybrid routing schemes [3] [5] [11] [14].

Scalability: This is defined by whether a routing scheme is scalable to the number of nodes in the network. In fact, most of the centralized routing schemes [7] [8] are not scalable because the computation for a large number of vehicle nodes can place a burden on a central controller. In contrast, most hybrid routing schemes [3] [5] [11] are scalable since the hierarchical architecture eliminates the workload of the central controller. In particular, vehicle clustering [3] is ideally scalable as it applies $\mathrm{CH}$ to organize the local networking issues while the network performance of the whole SDVN does not deteriorate much as the number of vehicle nodes increases.

Quality of Route: This refers to whether or not the data message follows an optimal route with low latency and high delivery ratio. Central routing schemes [7] [8] [14] provide highly efficient routes if they consider the realtime status of the vehicles in the computation of routes. Most hybrid routing schemes could not provide high quality routes, but not for the case of vehicular neighbor group (VNG) [11]. In VNG, the local controllers (i.e. RSUs) are only responsible for collecting regional status data. However, these controllers are not responsible for calculating routes while routes are mainly generated in the central controller based on real-time and historical data. Prediction-based routing schemes such as LDPR [13] calculate the routes based on the assumption that vehicles follow the optimal driving path in which this assumption could lead to generation of fail routes.

\section{Open Issues and Discussions}

The previous sections summarize the most representative routing schemes in SDVNs while also presenting comprehensive analysis. These approaches show good performance in terms of guaranteed delivery, routing overhead and so on. However, we still find a performance gap with the ideal routing approaches to fully utilize the advantages 
of SDVNs. In this section, we outline the open issues and opportunities for future research as follows.

Lack of Research in Dedicated Trajectory Prediction Algorithm: The trajectory prediction is to obtain the future status of the vehicle based on its current status. The traditional trajectory prediction algorithms normally apply different movement analysis models including Markov chain, Kinematic model, the Constant Speed Mobility (CSM) model, or Gauss-Markov Mobility (GMM) model and so on to predict the trajectory or location of vehicles. Plentiful historical data from the vehicle and its surrounding vehicles are applied by existing research. However, in SDVNs, it is vitally important to collect less data in order to avoid the burden on the controller. This leads to a new challenge: how to apply limited vehicle status data to predict vehicle trajectory in a short time horizon to meet the routing needs of SDVNs.

Further Reduction of Communication Overhead: The status beacons and routing messages sent from/to the controller generate high uplink/downlink communication overhead. Without assuming multiple networking interfaces in a vehicle, predicting vehicle trajectory in the controller is the top ranked solution for reducing the overhead. In this case, the amount of beacons status is greatly reduced while the controller can construct the network graph based on the collected status and the link predictions. Many related studies apply vehicle clustering in which only the $\mathrm{CH}$ communicates with the controller to reduce the overhead [14]. In this type of hybrid routing, $\mathrm{CH}$ normally acts as the local controller to collect the vehicle status in its dominant area and sends the cluster status and unsatisfactory routing request to the controller for further processing. The number of routing queries and status beacons are certainly reduced whereas the total size of status beacon data is not sufficiently lowered as the cluster status beacons are still sending. Even in the current hybrid SDVN, the central controller is still responsible for most multihop routing calculation. For future research, it is essential to enable the controller to distribute routing policies such as routing metric or specific routing schemes based on the network status to further reduce the communication overhead. Furthermore, it is worth constructing various models to evaluate the tradeoff between centralized and hybrid routing such as the routing mode of SDVN being adaptively switched under different network conditions.

Lack of Research in Routing Algorithms in Controller: Most existing works apply the static shortest path algorithm like Dijsktra, in the controller to compute the routes for routing queries. However, most links between vehicle pairs are only valid for a certain amount of time or the weight of the links between them are dynamic in SDVNs. Traditional shortest path algorithms for static networks are not applicable any more. Actually, this turns the routing problem into one of solving the fastest path in dynamic timetable graph. In fact, different research efforts considered the route planning problem in timetable graph in the database community [15]. Indeed, most of them focused on improving the online query efficiency of network pre-computations. In the SDVN environment, the controller could be extremely busy, handling millions of routing queries at the same time which demands that route planning algorithm are efficient enough to meet the networking requirements. Moreover, the existing algorithms are efficient, relying on pre-computations of the network which is impossible to achieve in dynamic SDVNs. Thus, as a future research direction, it is necessary to either import existing ones from road route planning or design an alternative to have an efficient timetable-dependent routing approach. Lack of Applying AI (Artificial Intelligence) in Routing Management in Controller: So far existing solutions lack the research of using $\mathrm{AI}$ to enhance the routing performance of SDVNs. In particular, exploring growing data traffic to manage network routing is a very promising approach to deal with dynamic and large-scale SDVNs. For example, statistical learning, neural networks or deep learning could be applied for constructing the routing metric, routing policy or even per-flow QoS (quality of service) parameters (latency, packet delivery ratio etc.) from the historical and real-time data. Despite the possibilities offered by the AI in SDVNs, a number of new challenges could also exist including computational complexity, cooperation of learning and manned strategy, and real-time data preprocess and so on.

Recovery Mechanism of Failed Routing Instructions: In practice, the route of data messages computed by the controller can be dropped when there is a broken link in the route due to the dynamic nature of the network. Unfortunately, heretofore existing studies do not present efficient route recovery mechanisms. The simplest way to repair a fail route is either initiating local recovery requests or sending the routing query to a controller for a new route. However, neither is a feasible way to completely fix the failure. In contrast, future failures can be avoided if we can trace back to find the hidden reason and fix it entirely.

Lack of Research in Multicast Routing: Most current studies focus on providing the unicast routing. However, multicasting is also a fundamental technology for many key vehicular applications such as collision avoidance, cooperative driving and so forth. It is vitally important to orchestrate the multicast decisions within the global-view controller to provide efficient one-to-many data dissemination. Lack of Considering Security in Routing: Security is a particular important issue in vehicular communications related to the safety of in-car passengers, vehicles, pedestrians and other public entities. With the existence of controller, SDVN could be less vulnerable to cyber-attacks than other types of wireless vehicular networks, by enabling the 


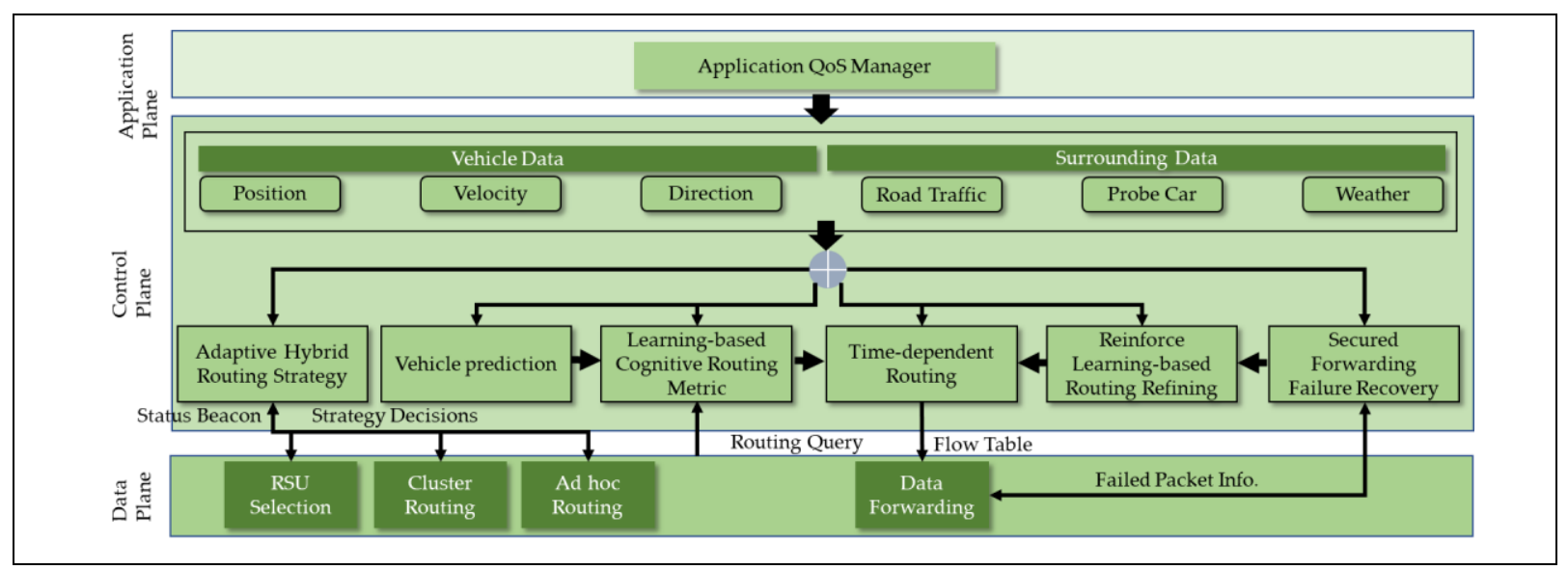

Figure 2. The routing management in SDVN

central coordination in controller. However, due to the nature of shared wireless medium in SDVNs, most conventional attack strategies could still work through injecting forged packets or changing the flow tables. The security for routing in SDVNs has not been well studied.

\section{Future Directions}

There is a strong case to study the framework of routing management illustrated in Fig. 2. In such framework, all types of route computation (unicast, multicast etc.) will be supported. To enhance the routing efficiency, the QoS parameters are assigned to the controller by the QoS manager of the application plane. Upon receiving these parameters, the controller learns the real-time and historical data from vehicles and surroundings to first draw up the routing schemes including the strategies of RSU selection, vehicle clustering rules and ad hoc routing policies. The schemes are then distributed to the vehicles to start up the interactions, uploading beacons or even local data forwarding. At the same time, the controller predicts the moving pattern of vehicles based on learning the data. While data cannot be forwarded locally or a controller switches the routing mode on the basis of the dynamic traffic flow, the routing queries are then sent to the controller. In this case, once the controller receives the routing query from a certain vehicle application, it constructs the network graph. This construction is based on the learned routing metric, the predictive links and the QoS parameter of that application, then sends the routes to the requesting vehicle. On receiving the reply, the vehicle starts forwarding data messages. When there is a failure during forwarding data messages, controller could use techniques such as negative provenance to trace back the causes and adopt reinforcement learning to refine the routing scheme. Further the routing schemes of SDVNs should consider the security issue during the whole procedures of routing to minimize the attacks and ensure the safety of vehicular networking. In the light of the above discussion, we find there is great potential and opportunities to improve the current routing schemes in SDVNs which can finally meet the communication requirements of future autonomous vehicle and vehicular services.

\section{ACKNOWLEDGMENT}

This work is supported by the National Science Foundation of China $(61701322,61502317)$ and the Key Projects of Liaoning Natural Science Foundation (20170540700).

\section{REFERENCES}

[1] B. Sharef, R. Alsaqour, and M. Ismail, "Vehicular communication ad hoc routing protocols: A survey," Journal of Network and Computer Applications, vol. 40, 2014, pp. 363-396.

[2] H. Saleet et al., "Region-Based Location-Service-Management Protocol for VANETs," IEEE Tran. Vehic. Tech., vol. 59, no. 2, 2010, pp. 917-931.

[3] S. Correia, A. Boukerche, and R. I. Meneguette, "An Architecture for Hierarchical Software-Defined Vehicular Networks," IEEE Commun. Mag., vol. 55, no. 7, 2017, pp. 80-86.

[4] M. Zhu et al., "SDN-Based Routing for Efficient Message Propagation in VANET," Proc. WASA. Springer International Publishing, 2015, pp. 788-797.

[5] E. Bozkaya and B. Canberk, "QoE-Based Flow Management in Software Defined Vehicular Networks," 2015 IEEE GC Wkshps, 2015.

[6] Z. He et al., "SDN Enabled High Performance Multicast in Vehicular Networks," Proc. IEEE VTC-Fall, 2016.

[7] Z. He, D. Zhang, and J. Liang, "Cost-Efficient Sensory Data Transmission in Heterogeneous Software-Defined Vehicular Networks," IEEE Sensors Journal, vol. 16, no. 20, 2016, pp. 7342-7354.

[8] M. Abolhasan et al., "Software-defined wireless networking: centralized, distributed, or hybrid?," IEEE Network, vol. 29, no. 4, pp. 32-38, 2015.

[9] Y. Peng et al., "A Novel Hybrid Routing Forwarding Algorithm in SDN Enabled Wireless Mesh Networks," Proc. IEEE HPCC, 2015, pp. 1806-1811.

[10] B. Dong et al., "Software Defined Networking Based On-Demand Routing Protocol in Vehicle Ad-Hoc Networks," ZTE Commun., vol. 15, no. 2, 2017, pp. 11-18.

[11] X. Huang et al., "Exploring Mobile Edge Computing for 5G-Enabled Software Defined Vehicular Networks," in IEEE Wire. Commun. Mag., vol. 24, no. 6, 2017, pp. 55-63.

[12] M. Zhu et al., "Efficient multiple-copy routing in software-defined vehicular networks," Proc. IEEE ICT 2015, Xi'an, 2015, pp. 1-6.

[13] K. L. K. Sudheer, M. Ma, and P. H. J. Chong, "Link Dynamics Based Packet Routing Framework for Software Defined Vehicular Networks," Proc. IEEE GLOBECOM, 2017. 
[14] H. Ghafoor and I. Koo, "CR-SDVN: A Cognitive Routing Protocol for Software-Defined Vehicular Networks," IEEE Sensors Journal, vol. 18, no. 4, 2018, pp. 1761-1772.

[15] S. Wang et al., "Efficient Route Planning on Public Transportation Networks: A Labelling Approach," ACM SIGMOD, 2015, pp. 967-982.

\section{BIOGRAPHIES}

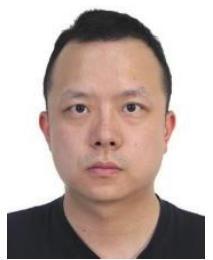

LIANG ZHAO (lzhao@sau.edu.cn) is a lecturer at Shenyang Aerospace University, China. He received his $\mathrm{PhD}$ degree in Computing from the School of Computing at Edinburgh Napier University in 2011. Before joining Shenyang Aerospace University, he worked as an associate senior researcher in Hitachi (China) Research and Development Corporation from 2012 to 2014. His research interests include VANETs, SDN and WMNs. His postal address is 37 Daoyi South Avenue, Shenbei New Area, Shenyang, China.

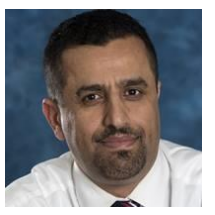

AHMED Y. AL-DUBAI [SM] (a.al-dubai@napier.ac.uk) is Professor of Networking and Communication Algorithms in the School of Computing at Edinburgh Napier University, UK. He received the $\mathrm{PhD}$ degree in Computing from the University of Glasgow in 2004. His research interests include Communication Algorithms, Mobile Communication, Internet of Things, and Future Internet. He received several international awards. His postal address is 10 Colinton Road, Edinburgh, United Kingdom.

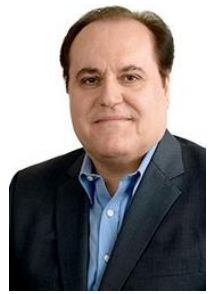

AlBERT Y. ZOMAYA [F] (albert.zomaya@sydney.edu.au) is a Chair Professor and director of the Centre for Distributed and High Performance Computing at the University of Sydney. He has published more than 500 scientific papers and is an author, co-author, or editor of more than 20 books. He is the Editor-inChief of IEEE Transactions on Sustainable Computing and serves as an Associate Editor for 22 leading journals. He is a Fellow of AAAS and IET. His address is University of Sydney, Sydney, Australia.

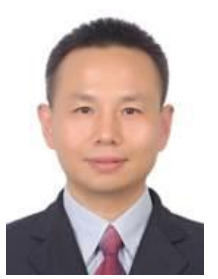

GEYONG MIN (g.min@exeter.ac.uk) is a professor of high-performance computing and networking in the Department of Mathematics and Computer Science within the College of Engineering, Mathematics and Physical Sciences at the University of Exeter, United Kingdom. He received his Ph.D. degree in computing science from the University of Glasgow, United Kingdom, in 2003, and his B.Sc. degree in computer science from Huazhong University of Science and Technology, China, in 1995. His research interests include future Internet, computer networks, wireless communications, multimedia systems, information security, high-performance computing, ubiquitous computing, modeling, and performance engineering. His postal address is University of Exeter, Exeter, United Kingdom.

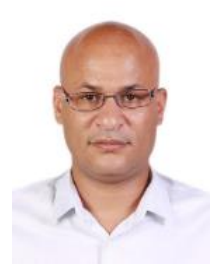

AMMAR HAWBANI (anmande@ustc.edu.cn) received the B.S., M.S. and Ph.D. degrees in Computer Software and Theory from the University of Science and Technology of China (USTC), Hefei, China, in 2009, 2012 and 2016, respectively. Currently, he is a postdoctoral researcher in the School of Computer Science and Technology at USTC. His research interests mainly in WSN and WBAN. His postal address is 96 JinZhai Road, Baohe District, Hefei, China.

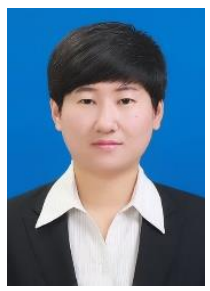

JIAJIA LI (lijiajia@sau.edu.cn) is a lecturer at Shenyang Aerospace University, China. She received the B.S. degree from Northeast Normal University in software in 2008 and received the M.S. and Ph.D. degrees in Computer Science from Northeastern University in 2010 and 2014. Her research interests include mobile data management, spatial-temporal database, intelligent transportation system. Her postal address is 37 Daoyi South Avenue, Shenbei New Area, Shenyang, China. 